\title{
通
}

\section{Estrés laboral o doble presencia en mujeres: abordaje desde la salud ocupacional}

Labor stress or double presence in women: its address from occupational health Angélica María Gavidia Pacheco*

* Abogada de la Universidad Simón Bolívar. Especialista en Derecho Penal y Criminología de la Universidad Libre. Magíster en Educación de la Universidad Simón Bolívar. Maestrante en Ciencias Políticas y Derecho Público de la Universidad de Zulia (Venezuela). Docente de la Universidad Nacional Abierta y a Distancia. Correo electrónico: ngelica.gavidia@ unad.edu.co 


\section{Introducción}

El papel de la mujer, históricamente, ha estado orientado hacia las labores del hogar, la atención y el cuidado de los miembros del grupo familiar; dicho papel se ha caracterizado por una marcada diferencia con respecto a su posición frente al hombre, destacándose por la sumisión y discriminación como pautas transmitidas de generación en generación. No obstante, a partir de los cambios ocurridos en la primera mitad del siglo XX se ha desplegado todo un proceso de transformación de esta situación, gracias a la lucha y a los movimientos de grupos feministas. En la actualidad, la población femenina ha ganado espacios en los ámbitos educativos, económicos, políticos, científicos, culturales, religiosos y sociales, a través del reconocimiento de sus derechos y garantías de igualdad de condiciones ante el hombre (Murguialday, 2006).

Hoy, el sexo femenino ha logrado incorporarse a espacios que eran destinados a los hombres, apoyadas en el desarrollo de políticas que -impulsadas por organismos internacionales - promueven la autonomía, libertad e independencia de la mujer, bajo los principios de equidad, igualdad y empoderamiento de esta frente al sexo masculino, con el fin de promover la calidad de vida del género femenino (Murguialday, 2006; Programa de Naciones Unidas para el Desarrollo, 1995).

Además de lo anterior, se presenta la necesidad de gran parte de la población de incorporarse al mercado laboral con el propósito de satisfacer las necesidades y demandas del grupo familiar, siendo la mujer, en la mayoría de los casos, la cabeza de familia que debe asumir esta responsabilidad ante la ausencia de la pareja u otro miembro familiar que contribuya con esta labor. En América Latina este hecho ha presentado un repunte desde inicios de este siglo, evidenciándose lo siguiente:

En los últimos diez años, la tasa de participación laboral de las mujeres en América Latina ha experimentado un crecimiento sostenible, dicha tasa ha tenido un incremento promedio de cerca de 10 puntos porcentuales, pasando del $45 \%$ a un $55 \%$ de la población femenina urbana de más de 15 años de edad; sin embargo, la tasa de actividad varía 
mucho de país en país; por ejemplo, en Argentina se ubica próxima al $60 \%$ y en el Perú, al 70\%, este hecho se debe a múltiples factores, como la privatización de los servicios públicos y nuevos patrones de consumo, que han ocasionado la necesidad de mayores ingresos y el aumento del número de "contribuyentes" en cada hogar. Esta es una tendencia que va en aumento. Además, el $48 \%$ de estas mujeres, son simultáneamente amas de casa y se "calcula que cumplen 16 horas de trabajo tanto doméstico como laboral, o sea que cumplen 112 horas semanales de trabajo cuando la mayoría de los códigos laborales del mundo, contemplan una jornada que oscila entre 40 y 45 horas semanales (Braña, 2008, p. 99).

Esta realidad se mantiene, pues en esta región la fuerza laboral femenina es altamente productiva para la economía del mundo y en general de los países (Datosmacro.com, 2014). En este mismo orden de ideas, Colombia presenta, para el primer semestre del 2017, una tasa global de participación laboral de las mujeres del $55,2 \%$; teniendo un incremento significativo con respecto al mismo periodo del año 2016, que fue del 48,4\% (Departamento Administrativo Nacional de Estadística, 2017).

Son múltiples los factores que llevan a la población femenina a incorporarse al mercado laboral, lo cual ha ocasionado la diversificación de su papel en la sociedad actual. Esto ha generado un cambio en la dinámica de la mujer dentro y fuera del hogar, pues se incrementan sus responsabilidades al tener que asumir dos o más jornadas, en las que se le exige ser competitiva y eficiente con respecto al hombre. Bajo este panorama, las mujeres requieren del despliegue de un conjunto de habilidades y destrezas que les permitan mantener un equilibrio entre ambas actuaciones (el hogar y la vida laboral); de lo contrario, se generan situaciones de desequilibrio, en virtud de las exigencias del medio y los recursos con los que se cuenta, que pueden producir ansiedad y tensión, promoviendo una patología que se conoce como estrés laboral o doble presencia, que afecta la salud física, emocional y relacional de este grupo social. 
A lo largo del tiempo se han desarrollado diferentes concepciones sobre el estrés, destacándose algunas de corte biologisista que se centran en las respuestas orgánicas; otras que plantean la naturaleza psicosocial a partir de factores internos y externos que lo generan, así como perspectivas más actuales integrales y sistémicas que destacan la interacción entre la persona y el ambiente. El estrés se considera como una patología porque afecta la salud física y mental de la persona, pero también tiene un componente de corte social debido a las consecuencias que genera en las relaciones familiares, laborales y sociales, que afectan el normal desempeño de las funciones diarias de quien lo padece (Aust). En el ámbito laboral, este se define como:

Un desequilibrio entre las exigencias ocupacionales y los recursos del entorno o personales con los que cuenta el trabajador, se presentan una serie de reacciones, que incluyen respuestas fisiológicas como; aumento del ritmo cardiaco o de la presión arterial, hiperventilación, así como secreción de las hormonas del "estrés" tales como adrenalina y cortisol); respuestas emocionales como: sentirse nervioso o irritado, respuestas cognitivas como: reducción o limitación en la atención y percepción, falta de memoria y reacciones conductuales como: agresividad, conductas impulsivas y errores constantes. (Organización Mundial de la Salud, 2002, p. 12).

El estrés laboral en mujeres posee una característica particular, a la cual se le ha denomınado la doble presencia, debido al "doble rol que juegan las mujeres como amas de casa y trabajadoras, producido por el hecho de la masiva incorporación de estas al campo laboral [...] esta situación no se produce en forma consciente en la mujer" (Balbo, 1978, p. 3). La sobre carga de actividades, dentro y fuera del hogar, influye en el deterioro de la calidad de vida de las mujeres, pues ocasiona un desgaste físico y mental que afecta otros ámbitos de sus vidas.

Son diversos los factores que propician la aparición del estrés laboral o doble presencia en mujeres, entre los que se encuentran algunas condiciones ocupacionales, por ejemplo, el trabajo directo con personas, en especial si estas tienen necesidades especiales, tales como: hospitalizados, enfermos 
mentales, ancianos, discapacitados, niños, niñas y adolescentes (Naranjo, 2009). En este análisis nos enfocaremos en las experiencias que vive el personal docente de las instituciones educativas, quienes a diario atienden a niñas y niños que demandan especial cuidado y atención.

En esta investigación nos interesa examinar la forma en que el estrés afecta la salud de los individuos; entendiendo la salud como "un estado de completo bienestar físico, mental y social, y no solamente la ausencia de afecciones o enfermedades [...]" (Organización Mundial de la Salud, 2006; p. 1). En consecuencia, se hace necesario el desarrollo de programas y proyectos dirigidos a abordar los factores generadores de este hecho en los diferentes contextos donde se desenvuelve el ser humano, destacándose en la actualidad los aportes desde el enfoque de la salud ocupacional dirigidos a atender el ámbito laboral.

Al respecto, se plantea que la salud ocupacional representa un conjunto de acciones multi y transdisciplinarias, cuyo propósito es controlar y prevenir cualquier factor que afecte la seguridad y la salud de los trabajadores. Además, plantea la necesidad de crear y promover ambientes saludables que puedan conducir a estilos de vida sociales y económicamente productivas, que fomenten el desarrollo humano y profesional (Organización Mundial de la Salud, 1995). El siguiente estudio examina las vivencias del estrés laboral o doble presencia en mujeres, con el fin de comprender esta realidad, para lo cual propone algunos lineamientos de intervención desarrollados por la institución educativa, abordados desde el enfoque de la salud ocupacional.

\section{Marco epistemológico y metodológico}

\section{Orientación epistemológica}

En todo proceso investigativo que esté orientado a dar respuestas a una situación problema, el investigador debe realizar una reflexión de carácter epistemológico, con la intención de darle rigurosidad a la misma, también debe preguntarse por la naturaleza del conocimiento que genera, su dependencia 
o independencia respecto al conocimiento producido, la interpretación que él hará de la realidad, así como el enfoque adecuado al objetivo de la investigación.

En este orden de ideas, cuando se plasman los tópicos sobre el conocimiento científico, se identifica primero la definición de conocer. Resaltando los postulados de los autores Tamayo y Tamayo, quienes exponen lo siguiente:

Conocer es enfrentar la realidad; todo conocimiento es forzosamente una relación en la cual aparecen dos elementos relacionados entre sí; uno cognoscente, llamado sujeto, y otro conocido, llamado objeto. Esta relación implica una actividad en el sujeto, la de aprehender el objeto, y la del objeto es simplemente la de ser aprehendido por el sujeto. (2013, p. 15).

Con el transcurrir de los tiempos el hombre manifiesta una diversidad de respuestas como ser humano, las cuales están condicionadas por la episteme.

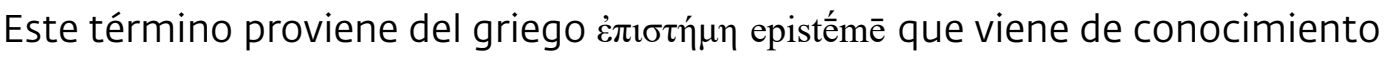
o ciencia. En lo concerniente, el espíteme, para cimentarse y sostener su andamio con estabilidad, se enfoca en dos verbos: "espítelo" elevar la columna y "epistano" mantenerse sobre, establecer o fijarse sobre. En virtud de lo anterior, la epistemología permite desarrollar disquisiciones de los contextos probables y la validez de hacer ciencia, lo cual busca excluir cuestiones concretas transitorias y particulares de la ciencia práctica. Como señala Padrón, "cuando los seres humanos producen conocimiento científico, trasladan a la esfera de la ciencia sus propios estilos de pensamiento, ahora convertidos en enfoques epistemológicos" (2013, p. 107).

Toda investigación inicia con el estudio de la epistemología, entendida como:

[...] ciencia o teorías de la ciencia; tiene por objeto conocer las cosas en su esencia y en sus causas. La epistemología presenta el conocimiento como el producto de la interacción del hombre son su medio. Conocimiento que implica un proceso crítico mediante el cual va or- 
ganizado el saber hasta llegar a sistematizarlo, como es el caso del conocimiento científico. (Tamayo, 2013, pp. 25-26).

Al delinear el conocimiento se está abordando el andamio de toda actividad social que realizan los hombres a diario, y que en todo momento está subordinado como una yuxtaposición a la diversidad de factores que la concurren. En sí, es el estudio la realidad natural, tal como se presenta para la investigación. Por consiguiente, la presente investigación se orientó en los fundamentos de la filosofía pos positivista, la cual postula el modelo dialéctico y sistémico para comprender la realidad que parte del supuesto de que el conocimiento es un resultado de la vinculación entre el sujeto investigador y el sujeto investigado. Este modelo plantea que el conocimiento es relativo, consensuado y parcial, circunscrito a un contexto social.

En efecto, "la epistemología pos positivista hace ver que no existe en el 'proceso cognoscitivo' de nuestra mente, una relación directa entre la imagen empírica, visual, auditiva, olfativa y la realidad externa [...] sino que siempre esta mediada e interpretada por el horizonte personal e individual del investigador [...]" (Martínez, citado por Del Canto, 2012, p.166).

\section{Postura metodológica}

Este estudio se circunscribió bajo el enfoque paradigmático de la investigación cualitativa, la cual se caracteriza por poseer un perfil abierto y flexible que orienta al investigador en temáticas relevantes que engloban el conjunto de prácticas de vida de los actores sociales. En efecto, así como lo sustenta a la luz del tejido investigativo, para Hernández, Fernández y Baptista, la investigación cualitativa:

Se enfoca en comprender los fenómenos, explorándolos desde las perspectivas de los participantes en un ambiente natural y en relación con su contexto. El enfoque cualitativo se selecciona cuando el propósito es examinar la forma en que los individuos perciben y experimentan 
los fenómenos que los rodean, profundizando en sus puntos de vista interpretaciones y significados. (2014, p. 358).

En este panorama investigativo, el enfoque pos positivista-cualitativo en sus definiciones devela un carácter interpretativo, constructivista y naturalista. De tal forma que le permite al investigador, al observar el contexto natural de la problemática, captar su comportamiento y todo aquel acontecimiento que prevalezca, para rediseñarlo y generar algunas teorías de acuerdo con los hallazgos encontrados, de allí su carácter inductivo. Sin embargo, "aunque el enfoque cualitativo es inductivo, necesitamos conocer con mayor profundidad el terreno que estamos pisando" (Hernández y Col, 2014, p. 358). Finalmente, la investigación cualitativa se enmarca en la comprensión de la realidad en su totalidad, lo cual significa que el horizonte de la cualidad del fenómeno estudiado se aprecia como un aspecto vinculado a un contexto global.

\section{Método de investigación}

La investigación cualitativa le brinda al investigador una diversidad de formas de distinguir e interpretar la realidad social. Por consiguiente, un proceso de investigación implica la realización de una serie de actividades para lograr un fin, lo cual nos remite ineludiblemente a un método. En este sentido, para este estudio se recurrió al método hermenéutico. La palabra hermenéutica proviene del vocablo griego hermeneuein, y su significado está referido a la interpretación, en cuyo caso el sentido auditivo pasa a ocupar un lugar importante en todo el proceso de investigación.

Uno de los principales exponentes del método Hermenéutico fue Dilthey que define la hermenéutica como "el proceso por medio del cual conocemos la vida psíquica con la ayuda de signos sensibles que son su manifestación". Esta definición implica que la hermenéutica permite descubrir los significados de las cosas, interpretar las palabras, los escritos, los textos y los gestos, conservando la singularidad en el contexto del que forma parte. Es un método de sistematización de 
procedimientos formales, en la ciencia de la correcta interpretación y comprensión. (Martínez, citado por De Canto, 2012, p. 195).

La hermenéutica parte del supuesto de que el ser humano es eminentemente interpretativo, por cuanto todo debe ser interpretado. Comprender es el proceso de construcción que conduce a elaborar la vida de otra persona, de tal forma que las interpretaciones producen otras interpretaciones.

\section{Informantes clave de la investigación}

La Institución Pública Educativa Distrital Sarid Arteta deVásquez (Barranquilla, Colombia) cuenta con una población de 54 docentes, conformados por 30 mujeres y 24 hombres. Con el objetivo de obtener una comprensión sobre el estrés laboral o doble presencia y su abordaje desde la salud ocupacional, para esta investigación se seleccionó al personal femenino, específicamente a 4 informantes clave: 2 docentes, la directora y la psicóloga de dicha institución.

\section{Recolección de la información}

Para el análisis e interpretación se tomaron en consideración algunas técnicas de investigación, las cuales guardaron correspondencia con el método hermenéutico. Entre ellas se seleccionó la entrevista semiestructurada mediante la cual se pudo conocer a los sujetos claves, sus vivencias conscientes e inconscientes y generar interpretaciones fehacientes de los significados contenidos en los fenómenos. Las entrevistas incluyeron la realización de una guía de preguntas vinculadas con el objetivo del estudio, con conversaciones abiertas y espontáneas, registrando estos datos en una grabadora, previo consentimiento de las personas entrevistadas y guardando fidelidad a lo expuesto.

Por otro lado, la transcripción, edición y categorización de las entrevistas conformaron el análisis de los dos procedimientos técnicos antes mencionados. 


\section{Interpretación, contrastación y teorización}

A continuación, se presentan los resultados obtenidos del proceso de análisis, contrastación e interpretación de las entrevistas realizadas a los sujetos involucrados en la realidad, en las cuales se exponen sus vivencias y opiniones, junto con los aportes que la investigadora realiza sobre la problemática del estrés laboral o doble presencia en las docentes de la Institución Educativa Distrital Sarid Arteta de Vásquez (Barranquilla, Colombia). Con el fin de indagar acerca del conocimiento que tiene el personal docente femenino de esta institución sobre el estrés laboral o doble presencia, se construyeron las siguientes categorías y subcategorías: definición, síntomas y factores o situaciones estresantes, las cuales se describen a continuación:

\section{Definición de estrés}

Para el desarrollo de este análisis se traen a colación las directrices emanadas del Informe de la Organización Internacional del Trabajo, en el cual se define el estrés como "la respuesta física y emocional a un daño causado por un desequilibrio entre las exigencias percibidas y los recursos y capacidades percibidos de un individuo para hacer frente a esas exigencias" (2016, p. 2). El término "estrés" fue utilizado por primera vez por Hans Selye, en 1936, quien lo definió, en términos biológicos, como"una respuesta inespecífica del cuerpo a cualquier solicitud de cambio". Actualmente, el término se utiliza para describir todo, desde sentirse enfermo por la mañana, hasta el estado de ansiedad que lleva a la depresión. Entre ciertos grupos científicos el estrés tiene connotaciones tanto positivas como negativas. El estrés puede referirse a una condición negativa o una condición positiva que responda a un factor estresante y que pueda tener un impacto en la salud mental o física y en el bienestar de una persona.

En este sentido, para González:

El estrés ha pasado a formar parte de nuestra vida cotidiana, comúnmente (con fundamento o sin él), se le asocia a inestables estados de 
ánimo y emocionales; de cansancio, de agotamiento físico/mental, de situaciones de tensión que en el trabajo cotidianamente enfrentamos individuos y colectivos enteros (obreros, oficinistas, profesionistas, en general trabajadores de las diferentes ramas industriales y de servicios tanto públicos como privados); lo que lo hace aparecer como el comodín, con la aptitud de reflejar una situación indeseable que el individuo deja ver en un crónico estado de nerviosismo y de ansiedad. Se trata de un círculo vicioso que al mismo tiempo parece ser la causa y el origen de ese malestar. El estrés, puerta de entrada y condición inherente al desgaste laboral de nuestro tiempo, goza de una gran popularidad, lo que a la larga ha terminado por condenarlo a una falta de especificidad, y en consecuencia no se le ha dado la importancia y el peso que realmente representa al momento de mirar las cada vez más documentadas repercusiones que tiene sobre la salud. (2012, p. 179-180).

Lo anterior se evidencia en las respuestas aportadas por las entrevistadas. Allí se puede señalar que existe un conocimiento general sobre este fenómeno, pues se identifica al estrés como parte de su vida. Esto tiene que ver con la respuesta fisiológica y psicológica del organismo ante alguna situación de sobrecarga laboral o afectiva, que simplemente emerge ante los diferentes eventos presentados durante el ejercicio de las actividades individuales, familiares, sociales y laborales. También se evidencian los factores externos e internos que este hecho presenta, incluso se considere este como un problema de salud en la actualidad que afecta la salud de la persona que la padece, siendo considerado una patología.

[...] Lo que más se asemeja a estrés es tensión, entonces se puede definir el estrés como un estado de tensión que vivimos diariamente en todas las situaciones de nuestra vida [...]. (Entrevista 1. Línea 23-29). [...] Para mí el estrés [...] es como el exceso de trabajo o la carga [...] la carga o el estado de ánimo que nos deja a nosotros el exceso de trabajo [...]. (Entrevista 1, Línea. 36-44).

[...] El estrés se ha venido definiendo como una patología [...]. (Entrevista 2, Línea. 12-15). 
[...] Es definido como una reacción fisiológica que se ejerce en el organismo, una vez que siente una amenaza de algún factor externo y que surge, pues este reacciona antes esos factores [...]. (Entrevista 4, Línea. 13-19).

[...] El estrés, como tal, serían todas esas situaciones que causan angustia al individuo, no siempre a nivel laboral sino a nivel familiar... (Entrevista 4, Línea. 21-28).

Con respecto al concepto de estrés laboral, se destaca que este constituye "la respuesta fisiológica, psicológica y de comportamiento de un individuo que intenta adaptarse y ajustarse a presiones internas y externas en el ámbito laboral" (Hoyo, 1996, p. 6). Sobre el estrés laboral se enuncia, además, lo plasmado por la Organización Mundial de la Salud:

El estrés laboral es la reacción que puede tener el individuo ante exigencias y presiones laborales que no se ajustan a sus conocimientos y capacidades, y que ponen a prueba su capacidad para afrontar la situación. Aunque el estrés puede producirse en situaciones laborales muy diversas, a menudo se agrava cuando el empleado siente que no recibe suficiente apoyo de sus supervisores y colegas, y cuando tiene un control limitado sobre su trabajo o la forma en que puede hacer frente a las exigencias y presiones laborales. $(2004$, p. 3).

Al respecto, las entrevistadas destacaron lo siguiente:

[...] El estrés laboral está relacionado con lo que te acabo de decir... la carga de responsabilidades a las cuales nos tenemos que enfrentar y todas las consecuencias que eso nos trae [...]. (Entrevista 1, Línea. 88-93).

[...] El estrés laboral todos los seres humanos tienen un estrés básico y general para responder a diferentes demandas del entorno. Pero cuando hablamos de estrés laboral, desde el punto de vista del distrés, nos referimos es a una condición física y mental que hace que la persona se 
sienta sobrecargada de demandas externas que puedan traer múltiples consecuencias desde aburrimiento a nivel laboral hasta enfermedades que pueden incapacitar a un trabajador [...]. (Entrevista 3, Línea. 29-42). [...] el estrés laboral son esas situaciones que surgen en el ámbito laboral y que causan angustia y ansiedad, que están precisamente ligadas a todo el ámbito de trabajo [...]. (Entrevista 4, Línea. 11-16).

En cuanto al abordaje de esta categoría, las entrevistadas destacan que el estrés laboral está relacionado con los elementos psicosociales o la sobrecarga de trabajo generada por las múltiples actividades que desarrollan. Alí la carga emocional y psicológica es muy alta debido al tipo de población que atienden, en comparación con los factores físicos que pudiesen existir. Una vez más se observa que, aunque la definición no está clara para las informantes, sí relacionan el estrés con su trabajo y con la carga de responsabilidades, las cuales salen a relucir en sus expresiones, es decir, al relatar sus experiencias personales, y no desde un conocimiento teórico del concepto de estrés laboral. De esta forma se puede resaltar que el estrés surge cuando se da un desajuste entre la persona, el puesto de trabajo y la propia organización.

La doble presencia se refiere a "la doble carga de trabajo que representa llevar a cabo un trabajo remunerado, como al doble origen, productivo y reproductivo, que define la identidad individual y social de las mujeres" (Almodóvar, 2008, p.4). Las entrevistadas definen la doble presencia de la siguiente forma:

[...] porque estoy realizando las dos cosas al mismo tiempo: sigo siendo ama de casa y llevo el trabajo para mi casa [... t tengo el trabajo y mis quehaceres. (Entrevista 1, Línea. 218-221).

[...] no es fácil, de verdad que no es para nada fácil cumplir, de forma individual, con las dos funciones: el de trabajadora y el de ama de casa [...] (Entrevista 1, Línea. 249-235).

[...] Mira, uno nunca debe generalizar... pero en función de lo que se ha dicho... sí se experimenta un fenómeno de doble presencia, de hecho, aquí es un trabajo arduo... exigen mucha dedicación personal, lo que, por supuesto, puede ser un potencial de estrés... y claro, son 
madres que no se escapan del fenómeno de la doble presencia porque llegan a su casa y tienen que cumplir labores de madre, de esposa... (Entrevista 3, Línea. 101-114).

De acuerdo con lo antes expuesto, se concluye que la doble presencia representa la omnipresencia de la mujer en todos los ámbitos de su vida, por cuanto desarrolla diversos roles dentro de la familia: esposa, madre, hermana, tía y abuela; no pudiendo, en muchos casos, desligarse de sus otros roles, los cuales la acompañan a donde quiera que vaya, bien sea del trabajo a la casa o viceversa, más aún cuando tienen hijos que atender y no cuentan con la ayuda de su pareja. Es así como la doble presencia es definida por las entrevistadas como una doble ausencia, pues produce en ellas la sensación de no atender plenamente ninguno de los dos ámbitos, lo que provoca frustración y estrés. Esta situación plantea una sobrecarga de trabajo, debido a que las mujeres llegan a sus casas agotadas de la actividad laboral y, sin embargo, deben cumplir con los quehaceres del hogar, disminuyendo el tiempo que podrían dedicar a su descanso, a los hijos o a la pareja.

Con respecto a los factores estresores, se destaca las diversas circunstancias de tipo físico o psicosocial que propician el estrés laboral o doble presencia (Pedrosa, et al., 2004 p. 32). Son muchas las situaciones que influyen para que el estrés aparezca en los individuos, entre las que se pueden mencionar el trabajo, la familia, el tráfico, la situación económica, los cambios, entre otros, (Braham, 1997). De esta forma,

El estrés en el entorno laboral es un problema creciente con un costo personal, social y económico notable. En toda situación, de estrés se da la responsabilidad de los directivos y de la propia organización en la medida en la que ésta lo causa o lo facilita, o en la medida en la que no lo detecta a tiempo o no lo remedia. Pero hay una responsabilidad ineludible también que reside en el propio empleado que es quien debe salir de esa situación, buscar asesoramiento, tomar decisiones difíciles y emprender cambios importantes en algunas parcelas de su vida, siendo a veces necesario incorporarse a un proceso terapéutico, 
no siempre fácil ni cómodo y a menudo prolongado. Todo ello requiere un esfuerzo y una perseverancia personal. (Sánchez, 2011, p. 16).

\section{Sumado a lo anterior, entre las entrevistas se destacan las siguientes: Personales}

[...] aparte de eso, son seres humanos que vienen de sus casas también cargadas de problemas personales. Es entonces difícil para nosotras atender a toda persona que presente situaciones depresivas (Entrevista 1, Línea 73-79). [...] siempre sale algo más importante, que dedicamos un poquito de tiempo a ese maltrato. Porque yo considero que es un maltrato lo que hacemos a nuestro organismo... yo misma me castigo con todo lo que sé que tengo que hacer... y que sé que existe la manera de distraerme, de lidiar con ese anti estrés y no lo hago... lo pongo siempre de segundo plano (Entrevista 1. Línea. 406-415). [...] Según la psicóloga, aparte de hiperactiva soy sobre activa. Parece que mi misma personalidad hace que me cargue de responsabilidades, entonces eso también genera estrés. (Entrevista 2, Línea. 112-118).

En estos testimonios, relacionados con el espacio personal, se visualiza la importancia de tener el autocontrol de la personalidad. Mantener una vida agitada en todo momento desfavorece y debilita la salud de las personas, incluso algunos médicos diagnostican esto como estrés. Es más, la carga más pesada de la vida, por lo general, la llevan las mujeres, pues un alto porcentaje de las familias a nivel mundial se caracterizan por ser matricentradas.

\section{Familiares}

[...] ellas no solamente están sometidas al estrés que hay en su casa con las situaciones económicas, las situaciones de los hijos, de pareja... (Entrevista 4. Línea 40-44). 
[...] ellas manifiestan situaciones que van desde la cotidianidad por la diversidad de roles que ejercen como madres, esposas, hijas. Por ejemplo, estar pendiente de los niños, de la casa, llevar a los hijos al colegio. Eso les genera un poco de estrés, el hecho mismo de estar pendiente de todo: su grupo familiar, su familia y todas las responsabilidades... (Entrevista 4, Línea. 72-84).

\section{Laborales}

[...] Por ejemplo, cuestiones en la escuela que hay que hacer de un día para otro con presiones, contra tiempo, con o sin recursos... (Entrevista 1 Línea. 131-126).

[...] Y las presiones laborales a las que tampoco nos podemos escapar. Y reconozco que me dejo absorber mucho por las presiones, me exaspero con las presiones laborales... (Entrevista 3, Línea. 106-111).

[...] esa mujer trabajadora, que tiene que ir todos los días, cumplir un horario, llegar a la casa, atender a los niños que van al colegio... (Entrevista 4, Línea. 108-112).

[...] es debido a la diversidad de situaciones de estrés que día a día acumulan, puesto que son madres, tienen que trabajar, tienen que salir a la calle todos los días y se va acumulando una tensión que va generando estrés... (Entrevista 4, Línea. 52-61).

Hace varias décadas que la mujer ha venido ocupando un rol de preeminencia como protagonista en el desempeño del área laboral; por ejemplo, como un sujeto de acompañamiento con el hombre, jefe de familia en los gastos de la economía familiar, etc. La gran mayoría de mujeres, luego de cumplir con su rol laboral, deben atender al núcleo familiar, mediante actividades como alimentar a sus hijos, cuidar a la pareja, sin contar con la ayuda de otros miembros de la familia. De esta forma, la mujer ejerce el porcentaje más alto de actividades, y en muchas ocasiones el total de los quehaceres y responsabilidades del hogar. 


\section{Participación política}

[...] uno va acumulando los compromisos del trabajo y de la casa. En este caso, como me pasaba a mí también, las responsabilidades políticas... (Entrevista 2 Línea. 33-39)

Me iba a las reuniones políticas, por decirlo así, que si el partido $X$ los sábados y domingos. Si había una jornada, una actividad o una concentración, ahí estaba yo metida en todo eso, entonces llegó un momento en el que estaba como ahogada dentro de ese mismo estrés... (Entrevista 2, Línea. 49-63).

\section{Multiplicidad de roles}

Hacer tantas actividades, al mismo tiempo, a veces no sale tan bien como uno espera, entonces eso es lo que yo considero que me genera bastante estrés, no el trabajo propiamente... (Entrevista 3, Lin. 136-142).

Afecta en la medida en que nosotras, como mujeres capaces de ejercer diversos roles, no sepamos enfrentar o canalizar esas emociones porque podemos ser mamas, podemos ser esposas, podemos ser trabajadoras, podemos estudia... (Entrevista 4, lin.149-156).

\section{Vivencias traumáticas}

Me impresionan mucho todas las situaciones sociales del país. No puedo ver noticiero porque me impresiono mucho cuando veo asesinatos, atracos... (Entrevista 3, Línea. 100-102).

[...] situaciones traumáticas que tienen que ver específicamente con robos, inclusive atracos, secuestros... (Entrevista 4, Línea. 88-91).

Lo anterior evidencia que las mujeres, en la actualidad, están expuestas a múltiples situaciones estresoras, producto de los diferentes roles que asumen. La 
incursión de la población femenina, cada vez en mayor número, a espacios que eran ocupados por el hombre, requiere que dividan su tiempo en la atención de cada una de estas actividades, concentrándose en el hogar y la actividad laboral; en otros planos se encuentran la participación política, comunitaria y social, que muchas veces consume su tiempo libre o descanso, afectando la atención hacia sus hijos, esposos o parejas. Aunado a esto, se presentan condiciones externas de tipo económico y de inseguridad que cada vez tienen mayor alcance en la ciudad, generando sentimientos de angustia y temor, agudizando los factores que llevan a la población femenina a experimentar las diferentes patologías que caracterizan al estrés.

El estrés laboral en mujeres, producto de la doble presencia, genera un conjunto de consecuencias físicas, familiares, laborales y sociales (Martínez, 2000). De igual forma, se destaca que este hecho da lugar a "serios trastornos personales, como: enfermedades digestivas, dermatológicas, cardiovasculares, respiratorias, inapetencia sexual, trastornos musculares, entre otros trastornos de la personalidad, o de la conducta que sin duda afectan las relaciones personales con sus grupos de pares y parejas" (Hoyo, 1996, p. 123). Al respecto, las entrevistadas destacaron:

\section{Personales}

Me siento cansada... que todo me molesta. No quiero que nadie me hable, siento que lo más importante... lo único, lo que yo tengo en ese momento en mi cabeza... no permito que la gente se me acerque porque estoy enfocada en lo que más me estresa... (Entrevista 1, Línea. 303-309).

[...] yo dormía poco, me alimentaba hasta mal porque se me pasaba la hora de comer por atender el trabajo... (Entrevista 2 Línea. 68-73)

[...] no dormía bien porque sacando trabajo de planificación de la escuela, de planificación de la universidad... (Entrevista 2, Línea. 81-85)

En vista de los nervios, mi vida social, por ejemplo, se ha truncado mucho... (Entrevista 2, Línea. 60-62). 
[...] se ha demostrado que esta doble presencia tiene efectos secundarios... Imagínate los altos índices por drogadicción sedante, son madres, que tiene niños pequeños, que trabajan... y además... son excelente madre, excelente esposa, aunque muchas veces las madres consumen un tipo de droga (Entrevista 3. Línea. 74-93).

[...] cuando estoy tranquila soy de una forma y cuando estoy estresada soy totalmente distinta... estoy distinta porque estoy reactiva ante las situaciones y ser reactivo no es bueno... (Entrevista 1. Línea. 678-684).

\section{Familiares}

[...] el hecho de llegar estresada... me duele la cabeza porque estoy cansada, me duelen las piernas por cualquier síntoma que yo pueda estar sintiendo. Entonces siento que mis hijos son responsables o que ellos son tan culpables, que los pongo a un lado... Cuando quiero descansar un poco, porque me siento preocupada, le digo a mi hijo: jay! Hijo, tengo un problema hoy y necesito que por favor me des un espacio más del que he durado todo el día en la calle...y entonces sí afecta muchísimo... (Entrevista 1, Línea 466-478).

[...] las consecuencias son divorcios, rupturas familiares, problemas con los hijos... Hemos tenido aquí a personas que llegan y tienen un hogar -que nosotros llamamos ejemplar- y la hemos visto divorciarse como consecuencia de las actividades que cumple en el trabajo, en las actividades políticas, lo que lleva a las personas a dejar a su familia en un segundo plano... (Entrevista 1, Línea. 154-166).

[...] como ya yo sé que voy a conseguir un reproche en mi casa; entonces ya yo me armo, me pongo, así como que voy a llegar formando lío para poder enfrentar, de alguna manera, la situación... Veo como preocupación de parte de ellos, mucha inseguridad. Me preguntan: "¿le paso algo a mi mama? No ha llegado a la casa". Y entonces yo siento que ellos me están reprochando... me están es exigiendo... porque de alguna manera me olvidé de aquel compromiso que tengo en la casa. (Entrevista 1, Línea. 445-462). 
[...] con mi pareja siento un poquito más de reclamo, más que con los niños... yo siento que él se pone muy celoso de mi trabajo, e incluso, cuando él me reclama, me dice cosas como "claro, porque tengo que decirte que es para tu trabajo, tu sales corriendo a buscarlo". O si me manda un mensaje, y yo no le respondo enseguida, "sí, claro como no es de tu trabajo" (Entrevista 2. Línea 246-257).

[...] Con relación a mi mamá, poco la veía, aun cuando estaba al lado. A veces me tenía que escribir para podernos comunicar por mensaje, solo así nos comunicábamos... (Entrevista 2, Línea. 146-157).

[...] recoger la casa que no pude recoger durante los cincos día de la semana, lavar la ropa, planchar la ropa, prepararlos para la jornada de la semana siguiente... (Entrevista 1. Línea 524 -529).

\section{Laborales}

Ese exceso de trabajo, ese ánimo cargado, no nos permite muchas veces cumplir con todas las funciones que debemos. (Entrevista 1, Lin. 37-42). [...] me siento cargada, me siento presionada y por cualquier situación me pongo reactiva... y entonces esto va en perjuicio también de mi rendimiento laboral. (Entrevista 1, Lin. 168-178).

El estrés laboral y la doble presencia afectan negativamente a la salud física y mental de las docentes, su relación con la familia (hijos, parejas o esposos, e incluso familia de origen), genera distanciamiento, conflictos, quejas, reproches, peleas constantes, problemas de comunicación e incluso rupturas relacionadas con sus roles laborales, políticos y sociales. En cuanto al ámbito laboral, se destacan las características emocionales y físicas que afectan su relación con los compañeros de trabajo y generan bajo rendimiento en su práctica laboral.

Finalmente, el análisis realizado sacó a relucir algunos lineamientos, en el marco de la salud ocupacional, que permiten abordar y prevenir el estrés laboral y la doble presencia en el personal docente femenino de la Institución Educativa Distrital Sarid Arteta de Vásquez. 
Con respecto a las estrategias institucionales, las entrevistadas señalaron lo siguiente:

[...]Se tendría que hacer un estudio organizacional, hacer un estudio transdisciplinario real para determinar qué es lo que le genera estrés a las mujeres, y en función de eso saber cuál es el foco generador de estrés..." (Entrevista 3, Lin. 172-179).

[...] el reconocimiento social de su trabajo, cuando lo hagan bien, y eso también serviría... (Entrevista 4, Lin. 167-169).

"Pienso que las labores siempreeee tienen que ser a nivel preventivo que es de lo que siempre carecemos, nosotros somos muy curativos cuando se presenta el problema es qué vamos y... pedimos atención...," (Entrevista 2 lin. 105-112).

...Buscar herramientas, porque el estrés nunca se va a acabar, el estrés es el motor de la vida, lo que tiene que evitarse son las consecuencias y cómo manejar esa situación de tensión para que a mí no me enferme... (Entrevista 1 lin. 126-133).

Mediante las estrategias organizacionales e institucionales, que emergieron de la narrativa de las entrevistadas, se evidencia la necesidad del reconocimiento social, a través del establecimiento de incentivos para el personal femenino que labora en esta institución. Esto último permite destacar la importancia de su labor y los múltiples roles que desempeña la mujer en la sociedad actual.

\section{Conclusiones}

La familia y la escuela constituyen los espacios más importantes para el desarrollo bio-psico social de los niños y niñas; en consecuencia, los padres y el personal que labora en las instituciones educativas son referentes de gran relevancia para esta población. Bajo esta perspectiva, el presente estudio se centró en "analizar el estrés laboral o la doble presencia y su abordaje desde la salud ocupacional en el personal femenino de la Institución Educativa Distrital Sarid 
Arteta de Vásquez de Barranquilla", desarrollado desde la investigación cualitativa, con el fin de profundizar en las dimensiones que tiene esta realidad.

Una vez concluido el presente estudio, se pudo evidenciar que la mujer actualmente desempeña múltiples roles como producto del avance de la sociedad y de las luchas de esta población por sus derechos, garantías e igualdad con respecto a los hombres. Así, las mujeres han venido ganando espacios en los contextos laborales, políticos, educativos y sociales, generando cambios en la dinámica familiar y social, teniendo que adaptar su tiempo a las diferentes exigencias de las actividades que desarrollan. Este hecho ha desencadenado diversas consecuencias, entre las que se encuentra el estrés laboral o doble presencia, que afecta la salud física y mental de este grupo social.

Con base en las entrevistas se pudo evidenciar que existe un conocimiento general sobre este fenómeno. Por ejemplo, las mujeres consultadas identificaron el estrés como parte de su vida; este se manifiesta a través de una respuesta fisiológica y psicológica del organismo, producto de las múltiples actividades que realizan en los diferentes ámbitos en que se desempeña. Esta situación se refleja en un conjunto de sintomatologías físicas y emocionales: dolores musculares fuertes, de cabeza, agotamiento físico, entre otros; acompañados de estallidos de ira, insomnio y depresión, los cuales a su vez desencadenan enfermedades como hipertensión arterial, cáncer, entre otras.

Con respecto a la doble presencia, esta se concibe como la doble jornada laboral que realizan las mujeres trabajadoras. De esta forma, las responsabilidades de la familia y las del ámbito laboral se cruzan, lo cual dificulta desligarse de los diferentes roles, más aún cuando tienen hijos que atender y no cuentan con la ayuda de su pareja. Esto hace que las mujeres experimenten una doble ausencia, al considerar que no están atendiendo adecuadamente ninguno de los dos ámbitos, lo que provoca frustración y agotamiento físico y mental.

Frente a los factores o situaciones estresores, se identificaron diversas situaciones que promueven esta patología, destacándose las de tipo personal (necesidad de la mujer de incursionar y participar en diferentes escenarios familiares, 
laborales, sociales, políticos y culturales), familiares (descuido de las funciones ante las exigencias de las actividades que desempeñan, demanda de los miembros de la familia, poco apoyo familiar), laborales (exceso de actividades, atención especial a los niños y niñas), participación política realizada en el tiempo libre, multiplicidad de roles simultáneos, económicas (necesidad de aportar al ingreso familiar o ser el soporte económico familiar) y las vivencias traumáticas (atracos, situaciones violentas, otros).

Algunas de las consecuencias que se derivan del estrés laboral o doble presencia tienen un carácter psico-social, relacionado con el deterioro de la salud física y mental, conflictos en las relaciones familiares, distanciamiento, separaciones, divorcios, bajo rendimiento laboral, entre otras. Finalmente se destaca la importancia de generar o fortalecer lineamientos estratégicos -en el marco de la salud ocupacional y en todos los espacios donde se desempeñan laboralmente las personas- que permitan abordar y prevenir el estrés laboral y la doble presencia en las mujeres.

\section{Referencias}

Almodóvar, M. (2008). Mujer y ciencia en Iberoamérica. Invisibilidad y familia. En Mujeres de ciencias: feminismo y ciencias naturales, experimentales y tecnológicas. España: Editorial Feminae, Universidad de Granada, Instituto de Estudios de la Mujer.

Balbo, L. (1978). La doppia presenza. En Inchiesta, núm. 32, pp. 3-11.

Braham, B. (1997). Cómo controlar el estrés y mantener la calma en situaciones difíciles. Santa Fe de Bogotá, Colombia: McGraw-Hill.

Braña, Y. (2008). Mujer y empleo en América Latina. En Punto de equilibrio, 17(99). Disponible en http://www.puntodeequilibrio.com.pe/punto_equilibrio/01i. phpantalla. [Consultado el 25 de agosto 2017] 
Datosmacro. Mercado laboral. Disponible en http://www.datosmacro.com/mercadolaboral. [Consultado el 25 de agosto 2017].

Del Canto, E. (2012). Investigación y métodos cualitativos: Un abordaje teórico desde un nuevo paradigma. En Revista ciencias de la educación. Segunda etapa, 22(40), pp. 181-199.

Departamento Administrativo Nacional de Estadística. (2017). Mercado laboral según sexo. Disponible en http://www.dane.gov.co/index.php/estadisticas-por-tema/ mercado-laboral/segun-sexo. [Consultado el 15 de agosto del 2017].

Enciclopedia de la Psicología. Sumario. Volumen 3. Editorial Océano

Hernández, R., Fernández, C. y Baptista, P. (2014) Metodología de la Investigación. México: Editorial McGraw Hill.

González, N. (2012). Estrés en el ámbito laboral de las instituciones de salud. Un acercamiento a las narrativas cotidianas. En Revista Argumentos, 25(70), pp. 171-192.

Hoyo Delgado, (1996): Estrés laboral. Revista de Seguridad e Higiene en el Trabajo, Volumen 40. pp (110-120). Madrid.

Martínez, Alfonso (2000). Calidad de vida laboral en función del estrés, tareas y antigüedad en trabajadores de una empresa hotelera. Tesis de Maestría en Psicología. Puebla. Universidad de las Américas. México.

Martínez, Miguel M. (2009). Ciencia y Arte en la Metodología Cualitativa. Métodos Hermenéuticos, Fenomenológicos y Etnográficos. Ed. Trillas. México.

Murguialday, Clara (2006). Empoderamiento de las Mujeres. Conceptualización y Estrategias.http://www.vitoriagasteiz.org/wb021/http/contenidosEstaticos/adjuntos/ es/16/23/51623.pdf. Recuperado el 20-06-2017. 
Naciones Unidas (1995). Informe sobre Desarrollo Humano. Programa de las Naciones Unidas para el Desarrollo (PNUD). México. Disponible en: http://hdr.undp.org/sites/ default/files/hdr_1995_es_completo_nostats.pdf. Consultado: 28 de mayo del 2017

Naranjo Pereira, María Luisa (2009). Una revisión teórica sobre el estrés y algunos aspectos relevantes de éste en el ámbito educativo. Educación, vol. 33, núm. 2. pp. 171-190 Universidad de Costa Rica San Pedro, Montes de Oca, Costa Rica.

Organización Mundial de la Salud (1995). Salud Ocupacional para Todos. Suiza-Ginebra (1995). http://apps.who.int/iris/bitstream/10665/42109/1/951802071X_spa.pdf (pág. 60. Párrafo 2) Consultado: 20 de mayo del 2017.

Organización Mundial de la Salud (2002). Programa Mundial de Acción en Salud Mental. Organización Mundial de la Salud. Disponible en: http://www.who.int/mental_ health/media/en/267.pdf. Consultado: 20 de mayo del 2017.

Organización Mundial de la Salud. (2004). La Organización del Trabajo y el Estrés. Serie Protección de Salud y los Trabajadores. N³. http://www.who.int/occupational_ health/publications/pwh3sp.pdf. Consultado: 29 septiembre 2017.

Organización Mundial de la Salud (2006). Constitución de la Organización Mundial de la Salud. Documentos básicos, suplemento de la 45a edición Disponible en: http://www. who.int/governance/eb/who_constitution_sp.pdf. Consultado: 20 de mayo del 2017.

Organización Internacional del Trabajo. (2016). Estrés en el Trabajo. Un Reto Colectivo. Primera Edición.http://www.ilo.org/wcmsp5/groups/public/---ed_protect/---protrav/--safework/documents/publication/wcms_466549.pdf. Consultado: 29 septiembre 2017.

Padrón, José. (2013) Epistemología Evolucionista: una Visión Integral. Edición Entretemas. 
Sánchez, Florencia. (2011). Estrés Laboral, Satisfacción en el Trabajo, y Bienestar

Psicológico en Trabajadores de una Industria Cereal era. Tesis para optar a título de Psicóloga. Universidad Abierta Interamericana.

Pedrosa, J.J.; Gaspar, J.; Dolz, J.L.; Barbolla, F.; Azzolini, R. (2004): Estrés postural. Ejercicios y mejora de hábitos. Zaragoza. Mira Editores.

Tamayo y Tamayo, M. (2013). El proceso de investigación científica. México. Editorial Limusa. 